\title{
Past climate changes and the role of refugia in the temperate Northern Hemisphere
}

Rachid Cheddadi and Keith D. Bennett ${ }^{2}$

\begin{abstract}
Glacial refugia in the temperate Northern Hemisphere proved to be havens for present ecosystems' diversity. The modern microrefugia, where isolated populations of threatened plant species are persisting, might well play a similar role for preserving future biodiversity.
\end{abstract}

\section{Classical and cryptic glacial refugia}

Plant species' potential range (fundamental niche) is delineated by a climate space that they often occupy only partially (realized niche). Generally, temperate plant species tend to occupy a wider geographical range when climate is warm and wet, and retract when climate is cold and dry. During the last glacial period, cold-tolerant herbs dominated the mid-to high-latitude landscape in both hemispheres and the range of most temperate tree species was reduced to discrete populations until the climate became warmer and wetter during the Holocene, which allowed them to expand.

The persistence or survival of plant species in restricted areas during past unfavorable climate time spans is a fundamental ecological pattern that has contributed to shaping the modern range of species, ecosystem composition, and species' genetic diversity. Restricted areas in the Northern Hemisphere such as in the Mediterranean basin, known today as "classical southern refugia" (Bennett and Provan 2008), played a major role in the survival of European plant species during the last glacial period. These geographically restricted and scattered areas located at lower latitudes offered local (micro) climates that were suitable for plant species. However, other refugial spots designated as "cryptic refugia" have been identified at higher latitudes than the classical southern refugia in the Northern Hemisphere, namely in Sweden (Parducci et al. 2012), Beringia, North America (Provan and Bennett 2008), and northern China (Fan et al. 2016).

Refugial areas where plant species were present continuously over the last climatic cycle under different extreme climates (Tzedakis et al. 2002), or since just the last glacial period until today under the modern fast climate change, are of primary importance for potential future species' conservation (Morelli et al. 2016). In southern Europe, scholars have shown that there is a strong relationship between the location of the classical glacial refugia and the modern areas where high biodiversity and endemism rates are measured (Fig. 1).

\section{Where are the refugia?}

Locating past refugia is also important for evaluating species' migration rates, hindcasting the species' range change through time using different climate scenarios, and testing and validating process-based vegetation models for competition between species. These species and/or vegetationtype models help to evaluate species' diversity loss under different future climate scenarios, their capacity for migrating, and the potential future suitable habitats for their conservation.

During a change in global climate, the areas where local microclimates are found to play the role of refugia for the persistence of plant species over several centuries or millennia (Tzedakis et al. 2002). This could be the case during either glacials or interglacials (Bennett and Provan 2008). Refugial areas are often located in mountainous regions (Bennett et al. 1991), which offer a wide range of landscapes, geological substrates, and exposure time to the sun. Mountains with high rugosity may provide more microrefugial areas (Rull 2009) for plant species under regionally less favorable climate than areas with smoother landscapes (Valencia et al. 2016). In addition, mountains represent a much faster option for species to compensate for global climate change by migrating easily up or down towards cooler/warmer and/or wetter/drier altitudes.

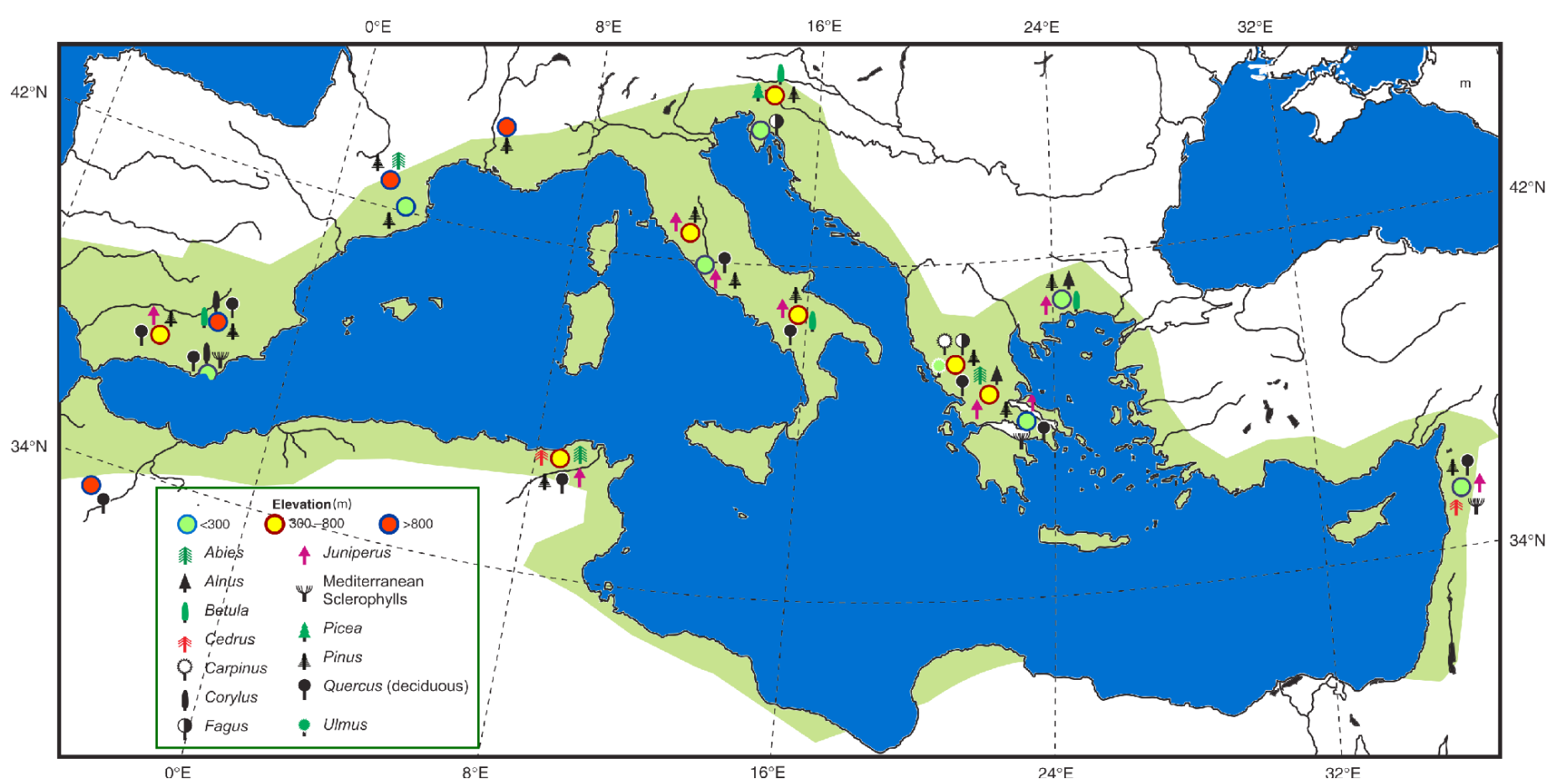

Figure 1: Inferred last glacial refugia (circles) of a few tree populations (symbols) from fossil pollen records collected from wetlands located at different elevations (modified from Tzedakis 2009). The green area represents roughly the present Mediterranean hotspot of biodiversity according to Myers et al. (2000; modified). Note that most of the identified glacial refugia are within the biodiversity hotspot. 

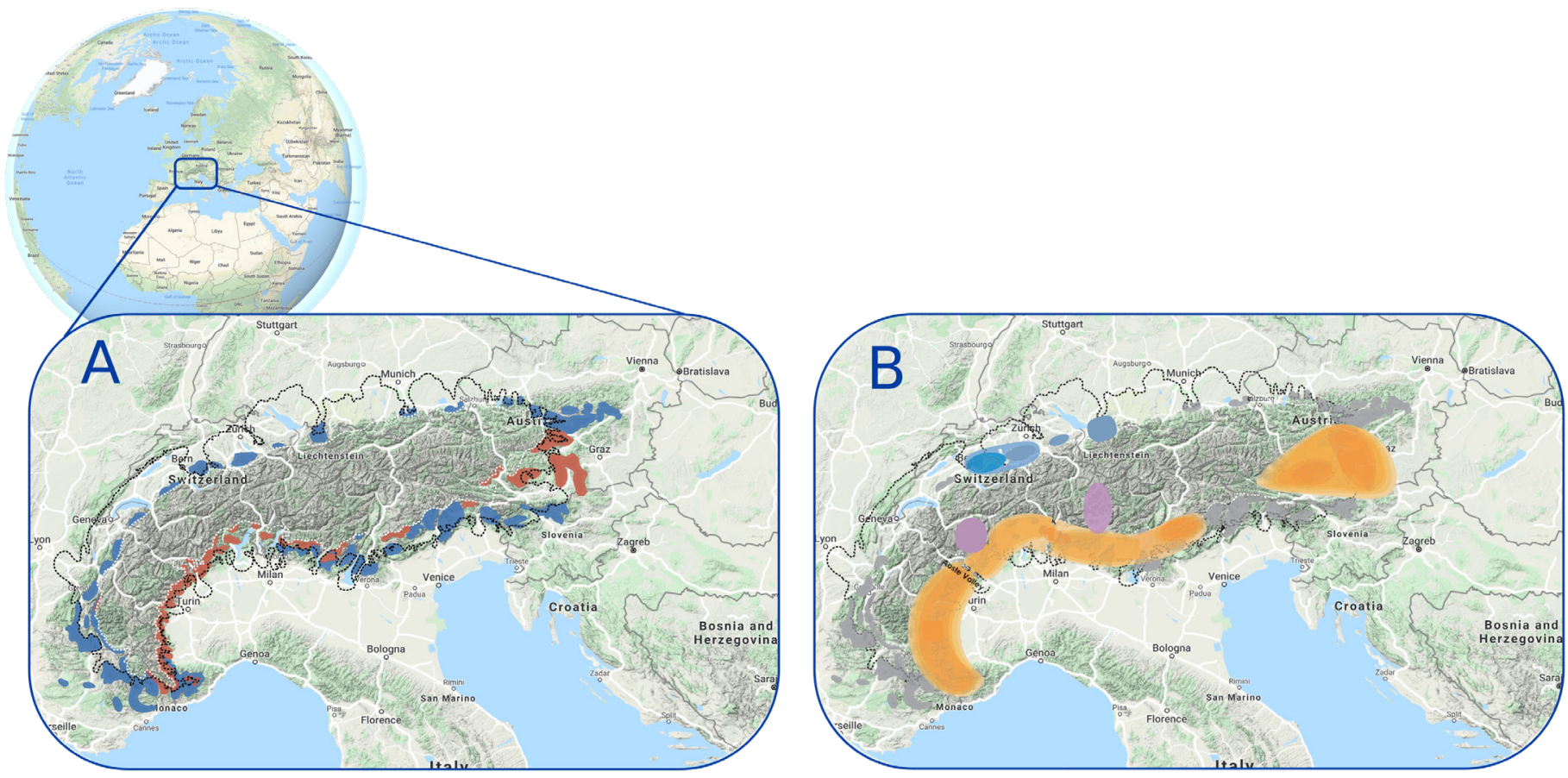

Figure 2: Multiple glacial microrefugia of different plant species around the Alps identified from (A) fossil records on different bedrocks (blue: calcareous; red: siliceous) that have been confirmed by (B) phylogeographic analyses, which provide more details about the different types of refugia (different colors on the map) and the species diversity that they shelter (modified from Schönswetter et al. 2005).

The Föhn winds can raise temperature and bring moisture from the adjacent seas to mountains, which may create local microclimates. By migrating $1000 \mathrm{~m}$ in altitude, a species may compensate for a temperature change between 6 and $10^{\circ} \mathrm{C}$, depending on the latitudinal location of the mountain (Bennett et al. 1991). Today, under ongoing global warming, many species persist as scattered populations in mountainous areas because of the existence of offset suitable microclimates. The estimated velocity of temperature change is lower within tropical and temperate mountain biomes than in lowlands (Loarie et al. 2009). Species respond individualistically to climate change, and their estimated past migratory rates seem to be slower than the modern climate change. Survival of many species under the ongoing climate change may rely on their ability to persist in some modern microrefugia (Rull 2009) with favorable (micro)climatic conditions.

\section{Glacial refugia and species' modern genetic diversity}

Refugial areas in the past can be identified from paleorecords and genetic data (Fig. 2). Fossil remains, such as pollen grains and macro-remains, allow for the identification of the occurrence of a species and its time of persistence at a site or the area surrounding the study site. Phylogeographers and paleoecologists have worked together over the past few decades to identify glacial refugia and to reconstruct the pathways of species expansion, based on species haplotypes using different genetic markers (Petit et al. 2003) along with extended sets of fossil pollen records.

For many species, microrefugia with stable microclimates that are offset from the global climate may represent the only alternative to extinction if their migration rate is lower than the velocity of climate change (Hannah et al. 2014). Modern microrefugia may have an important role in preserving species under the ongoing global climate change (Cheddadi et al. 2017) and may represent a reservoir of biodiversity. Genetic diversity would be maintained within these isolated populations and perhaps even improved if there is a genetic flow between different populations.

Phylogeographic studies have highlighted the role played by the glacial refugia for species survival and their legacy in shaping their modern genetic diversity (Petit et al. 2003). Depending on the range size and the time span over which plant species remain isolated in a refugium, there may be a local genetic shift of the species. The genetic drift during this time may lead to reduced genetic polymorphism in different geographically scattered refugia. Consequently, differences between DNA haplotypes could appear in the different refugia, which may be traced back by genotyping individuals of the same species collected over its modern range.

\section{Potential contribution of modern refugia to future biodiversity}

Genetic studies have shown that glacial refugia do not necessarily harbor the most diverse populations. However, the postglacial crossing of different lineages of the same species during the recolonization process from multiple refugia produced the most genetically diverse populations (Petit et al. 2003). Thus, even if the genetic diversity of a population tends to decrease over time when a species remains isolated in a refugium, its persistence into multiple scattered refugia may contribute to conserving and improving future species diversity. The future persistence of different species under the ongoing global warming and the preservation of biodiversity might well depend on the identification of potential modern refugia for threatened species and the protection of these refugia from anthropogenic degradation.

Identifying and protecting microrefugial areas may represent an additional option for species conservation (Hannah et al. 2014) which is likely to be one of the most efficient scientific solutions for species conservation with a reasonable social cost, especially in areas where local public resources are low.

\section{AFFILIATIONS}

IISEM, University of Montpellier, CNRS, IRD, France ${ }^{2} S$ chool of Geography \& Sustainable Development, University of St. Andrews, UK

\section{CONTACT}

Rachid Cheddadi: rachid.cheddadi@umontpellier.fr

REFERENCES

Bennett KD et al. (1991) J Biogeogr 18: 103-115

Bennett KD, Provan J (2008) Quat Sci Rev 27: 2449-2455

Cheddadi R et al. (2017) Front Ecol Evol 5: 1-15

Fan D et al. (2016) Sci Rep 6: 31044

Hannah L et al. (2014) Trends Ecol Evol 29: 390-397

Loarie S et al. (2009) Nature 462(7276): 1052-1055

Morelli TL et al. (2016) PLoS ONE 11: 1-17

Myers N et al. (2000) Nature 403: 853-859

Parducci L et al. (2012) Science 335: 1083-1086

Petit RJ et al. (2003) Science 300: 1563-1565

Provan J, Bennett KD (2008) Trends Ecol Evol 23: 564-571

Rull V (2009) J Biogeogr 36: 481-484

Schönswetter P et al. (2005) Mol Ecol 14: 3547-3555

Tzedakis PC et al. (2002) Science 297: 2044-2047

Tzedakis PC (2009) J Biogeogr 36: 1033-1034

Valencia BG et al. (2016) New Phytol 212: 510-522 